\title{
Madurella mycetomatis compounds cross-reactive with galactomannan are detectable in culture supernatant but not in serum
}

Madurella mycetomatis is a causative organism of eumycetoma, a disease characterized by tumorous masses, and formation of grains and purulent material (Ahmed et al., 2004). At present treatment of mycetoma is based on surgery and long-time antifungal therapy to prevent relapses. Ideally, continuous diagnostic monitoring of patients to determine the success or failure of antifungal therapy should be implemented. For the diagnosis of invasive aspergillosis, for instance, a sandwich ELISA is widely used (Mennink-Kersten et al., 2004). Circulating galactomannan or galactomannan-containing proteins are detected by the assay through an antibody raised against the galactofuran epitopes present on these molecules (Mennink-Kersten et al., 2004). Immunological cross-reactivity has been described for Paecilomyces variotii, Penicillium spp., Geotrichum capitatum and Cryptococcus neoformans (Dalle et al., 2005; Swanink et al., 1997). In the present study such galactomannan cross-reactivity was investigated for M. mycetomatis.

Culture supernatant from $32 \mathrm{M}$. mycetomatis isolates was obtained by culturing a $70 \%$ transmission inoculum in RPMI medium for 7 days at $37^{\circ} \mathrm{C}$ (van de Sande et al., 2005). Serum was obtained from patients and healthy controls, and frozen at $-20{ }^{\circ} \mathrm{C}$ until needed. The Platelia Aspergillus assay (Bio-Rad) was performed according to the manufacturer's instructions. The amount of galactomannan was determined from a concentration curve of pure galactomannan. Strain-dependent amounts of $M$. mycetomatis galactomannan-like compounds were secreted into the culture medium (Fig. 1). According to the cut-off value of $1 \mathrm{ng} \mathrm{ml}{ }^{-1}$, six isolates secreted no galactomannan-like compounds. The other 24 strains secreted these compounds in concentrations of up to $12.9 \mathrm{ng} \mathrm{ml}^{-1}$.

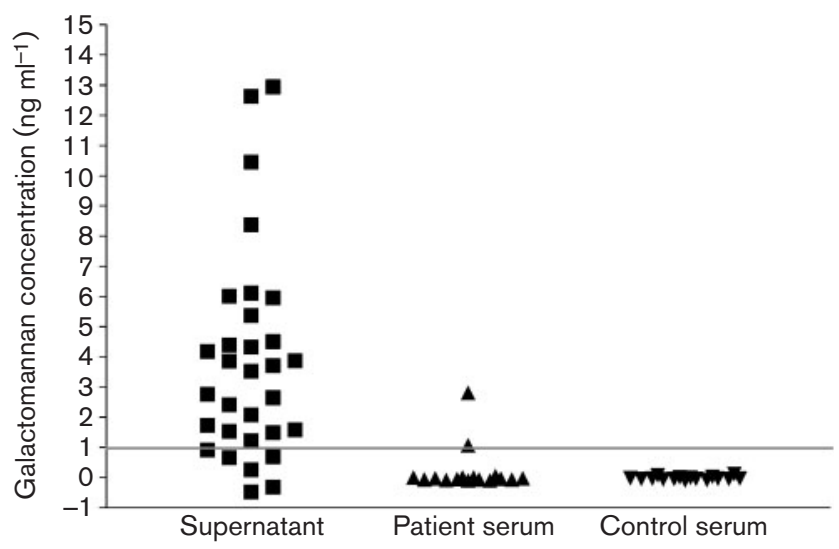

Fig. 1. Galactomannan concentration in M. mycetomatis. Galactomannan concentrations were determined in the culture supernatant after culturing for 7 days in RPMI culture medium $(\boldsymbol{\square})$. Galactomannan concentrations were also determined in patient serum $(\boldsymbol{\Delta})$ and serum from healthy Sudanese controls $(\boldsymbol{\nabla})$.

In only 2 of the 16 patient sera galactomannan levels above the cut-off value of $1 \mathrm{ng} \mathrm{ml}^{-1}$ were found (Fig. 1). There was no correlation with either the extent or the duration of the disease. In none of the healthy volunteer sera were galactomannan levels above $1 \mathrm{ng} \mathrm{ml}^{-1}$ found.

Significant amounts of galactomannan-like compounds were found to be secreted into the culture medium. Cross-reactivity in the galactomannan assay has already been described (Dalle et al., 2005). The fact that galactomannan is secreted in the culture medium but not recovered from patient circulation could be due to a variety of reasons. It could be that the structure of the sclerotia in patients (cement material, melanin, tight package) prohibits diffusion of the galactomannan. For aspergillosis it has already been demonstrated that in some cases galactomannan can be detected in the bronchoalveolar lavage but not in the serum (Becker et al., 2003; Verweij et al., 2000). Furthermore, it could be that antifungal treatment of mycetoma hampers the performance of this test. All patients were treated with either high doses of itraconazole or ketoconazole. Marr et al. (2005) demonstrated that administration of antifungal agents on the day of test sampling decreased the sensitivity of the assay significantly. It could also be that the antigen is not secreted because of a lack of nutrients. It has already been shown for Aspergillus niger and Penicillium fellutanum that secreted antigens can be reused as a carbon source when the organisms are deprived of nutrients (Mennink-Kersten et al., 2004; Park et al., 1997). In nutrient-rich conditions, Aspergillus spp. release high amounts of galactomannan during growth, but these amounts can be reduced in a nutrient-poor environment such as serum. In conclusion, $M$. mycetomatis secreted compounds that are immunologically cross-reactive to 
Aspergillus galactomannan in vitro but not in vivo.

\section{Wendy W. J. van de Sande, ${ }^{1}$ Ahmed H. Fahal, ${ }^{2}$ Henri Verbrugh ${ }^{1}$ and Alex van Belkum ${ }^{1}$}

${ }^{1}$ Erasmus MC, University Medical Center Rotterdam, Department of Medical Microbiology \& Infectious Diseases, Dr Molewaterplein 40, 3015 GD Rotterdam, The Netherlands

${ }^{2}$ Mycetoma Research Group, University of Khartoum, Khartoum, Sudan

\section{Correspondence}

Wendy W. J. van de Sande

w.vandesande@erasmusmc.nl

Ahmed, A. O., van Leeuwen, W., Fahal, A., van de Sande, W., Verbrugh, H. \& van Belkum, A. (2004). Mycetoma caused by Madurella mycetomatis: a neglected infectious burden. Lancet Infect Dis 4, 566-574.
Becker, M. J., Lugtenburg, E. J., Cornelissen, J. J., Van Der Schee, C., Hoogsteden, H. C. \& De Marie, S. (2003). Galactomannan detection in computerized tomography-based bronchoalveolar lavage fluid and serum in haematological patients at risk for invasive pulmonary aspergillosis. Br J Haematol 121, 448-457.

Dalle, F., Charles, P. E., Blanc, K., Caillot, D., Chavanet, P., Dromer, F. \& Bonnin, A. (2005). Cryptococcus neoformans galactoxylomannan contains an epitope(s) that is cross-reactive with Aspergillus galactomannan. J Clin Microbiol 43, 2929-2931.

Marr, K. A., Laverdiere, M., Gugel, A. \& Leisenring, W. (2005). Antifungal therapy decreases sensitivity of the Aspergillus galactomannan enzyme immunoassay. Clin Infect Dis 40, 1762-1769.

Mennink-Kersten, M. A., Donnelly, J. P. \& Verweij, P. E. (2004). Detection of circulating galactomannan for the diagnosis and management of invasive aspergillosis. Lancet Infect Dis 4, 349-357.

Park, Y. I., Buszko, M. L. \& Gander, J. E. (1997). Utilization of phosphocholine from extracellular complex polysaccharide as a source of cytoplasmic choline derivatives in Penicillium fellutanum. J Bacteriol 179, 1186-1192.

Swanink, C. M., Meis, J. F., Rijs, A. J., Donnelly, J. P. \& Verweij, P. E. (1997). Specificity of a sandwich enzyme-linked immunosorbent assay for detecting Aspergillus galactomannan. J Clin Microbiol 35, 257-260.

van de Sande, W. W., Luijendijk, A., Ahmed, A. O., Bakker-Woudenberg, I. A. \& van Belkum, A. (2005). Testing of the in vitro susceptibilities of Madurella mycetomatis to six antifungal agents by using the sensititre system in comparison with a viability-based 2,3-bis(2-methoxy-4-nitro-5-sulfophenyl)-5[(phenylamino)carbonyl]-2H-tetrazolium hydroxide (XTT) assay and a modified NCCLS method. Antimicrob Agents Chemother 49, 1364 1368.

Verweij, P. E., Weemaes, C. M., Curfs, J. H., Bretagne, S. \& Meis, J. F. (2000). Failure to detect circulating Aspergillus markers in a patient with chronic granulomatous disease and invasive aspergillosis. J Clin Microbiol 38, 39003901 